\title{
ADVANCED RADIATION DETECTOR DEVELOPMENT
}

\author{
Annual Progress Report \\ for the period February 17, 1998 to February 16, 1999
}

(Grant Year 1)

\begin{abstract}
Prepared for
THE U. S. DEPARTMENT OF ENERGY

AWARD No. DE-FG08-98NV13357
\end{abstract}

\author{
Glenn F. Knoll \\ Nuclear Engineering \& Radiological Sciences \\ College of Engineering \\ University of Michigan \\ Ann Arbor, Michigan 48109
}

February 16, 1999

Revision September 14, 1999 


\section{Introduction}

We have made substantial progress toward the major objective of this project: development of room temperature gamma-ray spectrometers with $2 \%$ or better energy resolution at $662 \mathrm{keV}$ fabricated from cadmium zinc telluride (CZT). Our principal source of material has been $\mathrm{eV}$ Products, but we are starting to explore alternate suppliers of the material. These include IMARAD Corporation in Israel, and Yinnel Tech, Inc. in South Bend, Indiana. These latter two sources are just beginning production using techniques that are alternatives to the traditional high pressure Bridgeman technique, and there are initial indications that the quality of material attainable may be better suited for detector fabrication than that currently available on the market. We have also begun to explore using cadmium telluride crystals that are available in larger size than CZT. In the past, CdTe has been limited by its relatively low resistivity that results in a high leakage current when conventional electrodes are used. However, we feel that this limitation can be overcome through the use of pixelated electrodes described later in this report.

We have taken the view that progress in attaining useful room temperature spectrometers from CZT can be pursued in two ways. One is the traditional approach of making the material better. This effort is laudable, but gains are gradual and hard-won. The other approach is the one we have undertaken of finding electronic methods to overcome the limitations of presently-available material. We first adopted the coplanar grid approach ${ }^{1}$ that Paul Luke of Lawrence Berkeley National Laboratory introduced, made improvements in the grid design, and currently hold the world's record energy resolution for CZT spectrometers of 1 cubic centimeter size. This achievement was made possible when we invented a unique technique for CZT depth-sensing that allowed us to electronically slice the detector into 20 separate layers. In a sense, each of these layers can be considered to be an independent detector, and we apply electronic corrections to make their responses as uniform as possible. Our technique of depth sensing is now a standard used by many other groups working with semiconductor spectrometers.

During the past year, we have made a major breakthrough in extending this approach to three dimensional position sensing. We successfully fabricated two 1 cubic centimeter spectrometers (out of two tries) that provide a full three-dimensional readout. Now we have the equivalent of 2,400 independent detectors, each with a volume of under 1 cubic millimeter. This allows us to accommodate changes in the material properties not only as a function of depth, but also in the other two dimensions. We see outstanding energy resolution from each individual voxel, and combine their outputs optimally for exceptional performance from the detector as a whole. This approach allows us to use only selected volumes which yield the best energy resolution, or the entire detector for maximum efficiency.

Our efforts have focused in three general areas:

1. Coplanar electrode configurations

2. Geometrically weighted, external grid devices

3. Pixellated and three-dimensional spectrometers 
Our general philosophy in pursuing good results from CZT recognizes that it is the transport of holes that is the real problem. Hole mobility is very low, and currently available materials show excessive trapping that prevents the clean collection of the charges represented by the holes. For these reasons, we have concentrated on configurations that do not rely on the motion of the holes, but generate a signal exclusively on the motion of the electrons. We feel that these so-called "single polarity charge sensing" devices have the greatest potential for producing viable spectrometers in the near future.

Despite advances in crystal growth technology, we feel that the nonuniformity present in large crystals of CZT will continue to be a potential problem for the foreseeable future. It is our observation that these nonuniformities exist not only as a function of depth into the crystal, but also across the entrance area as well. We have been the first group to demonstrate the successful correction for these nonuniformities as a function of depth, and are developing configurations that are also capable of corrections throughout the full three-dimensional volume.

While we support the efforts of others to improve the basic material through studies correlating impurities and/or crystal defects with charge transport, we feel that it is unlikely that any major breakthroughs will occur with this approach. The best that can be anticipated will be a steady but slow progress in improving the quality of the material. In contrast, there have been some major new capabilities made available within the last several years in the electronics that one can choose to process the signals from these detectors. We feel that exploiting these rapid developments in microelectronics is a very promising alternative to overcome the materials problems that persist. The solutions that are electronically complex today will become simpler in the future through the development of commercial Application Specific Integrated Circuits that incorporate many pulse processing functions in multiple channels on a single chip. We have been collaborating with IDE corporation in Oslo, Norway, in the development of the next generation of these chips, and feel that this is an exciting new frontier in providing capabilities that were not possible just several years ago.

\section{Major Accomplishments To Date}

1. We were the first group to introduce the concept of depth sensing in CZT detectors that is now standard practice in other laboratories carrying out CZT detector development. This is a major step forward since it allows for direct correction for the effects of crystal nonuniformities as a function of depth into the crystal. Furthermore, the depth-separated energy spectra that we are able to record shed light on the behavior of the material and have proven to be a valuable diagnostic tool.

2. Using a coplanar grid configuration, we have demonstrated a 1.8\% energy resolution (measured at 662 $\mathrm{keV}$ ) in a $1 \mathrm{~cm}^{3}$ coplanar spectrometer, which is the best reported for a crystal of this size to date. The development of the depth-dependent correction for trapping mentioned above was essential in achieving this result. 
3. We have been the first group to successfully demonstrate true three-dimensional sensitivity in a semiconductor spectrometer. This work is truly a breakthrough in the field since there have been no such threedimensionally resolved spectrometers previously demonstrated in any semiconductor material, including silicon and germanium. By exploiting the newly available readout chips from IDE, and combining a pixellated electrode pattern with our depth-sensing techniques, we are able to create a matrix of 2,400 voxels throughout the full volume of the detector. The output of each of these voxels is recorded separately, and we accumulate pulse height spectra independently for each. By measuring the variation in apparent gain throughout the entire volume, a set of correction factors is developed that can be used to overcome much of the effect of material nonuniformity throughout the crystal. Our first results on two such crystals show an energy resolution of $1.7 \%$ taken from the entire volume of the detector. Furthermore, this figure is now limited by the electronic noise level present in our readout chip. There are obvious ways to improve this readout noise, and we are pursuing improvements that will be present in the next generation of the ASIC currently under development in a joint project with IDE.

The complexity of these devices and the electronics required to support them follows the following hierarchy:

1. The geometrically weighted external grid detectors are simple and require only a conventional single preamplifier for their readout. This simplicity lends itself to ease of application in field use and keeps the cost of the device to a minimum. Furthermore, it is apparent that individual modules can be ganged together into larger volume spectrometers using signal combination techniques that are proven in other fields of gamma spectroscopy.

2. The readout of the coplanar electrode detector requires a differential preamplifier and a ratio circuit to enable the depth-dependent correction that is important in achieving the best energy resolution. Thus there is an added electronic complexity beyond that of a simple spectrometer, but the results make it clear that this addition greatly improves the energy resolution compared with a conventional detector readout.

3. The three-dimensional pixellated spectrometer requires the ASIC to be coupled to the detector, now accomplished using wire bond connections. We also require a more complex set of digital corrections to apply to the signals derived from all of the voxels, so this approach is the most complex from a signal processing standpoint. In its present form, it would not be suitable for a field instrument. However once the principle is established, it is easy to imagine more advanced versions in which the ASIC is mated to the pixellated electrode pattern through the use of bump bonding that is commonplace in the high energy physics detector community. This would lead to considerable simplification of the physical layout and take a major step toward having a totally integrated detector and readout package. Considering the rapid advancements that have been made in 
microelectronics in other fields, it is not unreasonable to expect that capabilities that are at the cutting edge today will become commonplace in the near future.

This approach would have been unthinkable just several years ago because of the complexity required in the electronics. However, newly-available multichannel ASICs have changed the picture almost overnight. On a chip that is no larger than one face of the detector, we currently employ 121 independent channels of readout electronics coupled to an 11 by 11 array of pixels. Our previously developed depth-sensing technique then provides the third dimension. To my knowledge, this is the first successful demonstration of a 3-D readout from any semiconductor detector, including silicon and germanium. We have already surpassed the performance of our best coplanar spectrometer, and are still far from having an optimized system. By being in the position of benefiting from the spectacular rate of development in microelectronics, progress has been much swifter than waiting for improvements in the material. Note that we are also not limiting our attention to CZT, but feel that our approach should also work well with CdTe.

Conventional CdTe spectrometers are available in larger volumes than CZT, but suffer from a much higher leakage current. But in a pixelated design, the leakage per small pixel is several orders of magnitude lower. We are therefore planning to evaluate the same approach on a 6 cubic centimeter CdTe crystal available from Japan if our funding constraints allow. This will be, if we succeed, the largest room temperature semiconductor spectrometer ever made.

\section{Other Technical Collaborations}

1. We are currently participants with GE Research laboratory, Schenectady, in a Small Business Innovative Research project on silicon avalanche photodiode development. This involvement came about through a trip by Professor Knoll to the Schenectady laboratories in 1997, and involves a proprietary GE process for the fabrication of arrays of avalanche photodiodes. Our role will be to evaluate these photodiodes as potential readout devices for scintillation detectors if the project succeeds in advancing to Phase 2.

2. We have also been collaborating with IDE in Oslo in specifying the design of the next generation of VA/TA readout chip that we will use with our three-dimensional CZT spectrometers. This has been a particularly fruitful collaboration, and we are very pleased with the level of cooperation we have received from Einar Nigard and his colleagues in the company. They have been very responsive to our particular needs, and have made several visits to our campus to help focus on the aspects of the design that are most critical for our applications.

3. We are in close communication with Dr. Kelvin Lynn of Washington State University in his development of novel techniques for producing patterned electrodes on CZT. Both the coplanar and the pixellated devices require such patterning, and Professor Lynn is developing a unique process that could make this step much 
simpler than with current methods. We are encouraged by his progress, and will be in a position to test coplanar grid patterns that are being fabricated in his laboratory when they are completed.

4. We continue to work closely with Dr. Carl Stahle of NASA Goddard Space Flight Center on the processing and contacting steps for large CZT crystals. He is particularly interested in applications of the 3-D configurations in a number of applications in gamma ray astronomy.

5. The project has continued to support the participation of Professor Knoll in meetings of the Radiation Detector Panel of DOE NN-20.

\section{REFERENCES}

1. P. N. Luke, IEEE Trans Nucl Sci 42, 207 (1995). 